\title{
Situation Assessment and Decision Making Strategies of Emergency Medical Services Physicians in Routine and Non-Routine Situations
}

\author{
Martin Sedlár \\ Institute of Experimental Psychology \\ Center of Social and Psychological Sciences, Slovak Academy of Sciences
}

\begin{abstract}
The aim of the study was to identify situation assessment and decision making strategies in emergency medical services physicians and to determine whether their distribution is associated with routine and non-routine situations. The research sample included 15 physicians, who were interviewed about routine and non-routine situations from their practice. The interviews about non-routine situations were conducted using the Critical Decision Method, and its adapted version was created for the purpose of conducting the interviews about routine situations. Generally, qualitative analysis showed that intuitive strategies were most frequently used in situation assessment and decision making but they occurred more in routine situations, by contrast, deliberative strategies were used more in non-routine situations. These findings were supported by statistical tests suggesting that physicians were more likely to change strategies with respect to situational demands.
\end{abstract}

Key words: situation assessment, decision making, emergency medical services, physicians, routine situations, non-routine situations, critical decision method

Naturalistic decision making (NDM) research is focused on macrocognition - cognitive functions and processes used by experienced people working in real-world settings (Crandall, Klein, \& Hoffman, 2006). These settings characterize ill-structured problems; uncertain dynamic environments; shifting, ill-defined, or competing goals; action/feedback loops; time stress; high stakes; multiple players; and organizational goals and norms (Orasanu \& Connoly, 1993). Exactly such setting, with multifactorial influences on cognition, is health care. In this field

This work was supported by the Slovak Research and Development Agency under the contract No. APVV0361-12.

Correspondence concerning this paper should be addressed to Martin Sedlár, Institute of Experimental Psychology, Center of Social and Psychological Sciences, Slovak Academy of Sciences, Dúbravská cesta 9, 84104 Bratislava, Slovak Republic. E-mail: martin.sedlar@savba.sk

Received November 15, 2016 evidence based medicine (EBM; Rosenberg \& Donald, 1995) is preferred, representing the normative approach, sometimes difficult to meet in real-world situations. By contrast, the main idea of NDM is that professionals rely heavily on their previous experience stored in memory, as described by the Recognition-Primed Decision model (RPD model; Klein, 1997). It is viewed as the experiential basis of intuition that enables rapid recognition of situations and decisions. Simply put, intuition is recognition (Simon, 1992).

Two of the primary macrocognitive functions are situation assessment and decision making (Klein et al., 2003), included in the RPD model. Situation assessment (SA) is about understanding of what is going on in the course of dynamic unfolding situations, during which elaboration of an existing SA or shifting to a new SA may occur repeatedly (Kaempf, Wolf, \& Miller, 1993). From the SA viewpoint, clear situations are usually immediately identified (recognized), and ambiguous situations need to be diag- 
nosed. In NDM, the term diagnosing has not been used only in a medical context, it has been used to express deliberative SA regarding different situational problems as well. Based on the review (Zsambok, Beach, \& Klein, 1992), there are the following SA strategies included in the RPD model: pattern matching - is about matching larger observed patterns with remembered patterns of previous situations; feature matching - is about matching observed features with remembered features of previous situations; and mental simulation - is about imagining the likely causal chain of events that preceded the observed situation. Adopting the theory of two processes (Evans, 2007), pattern matching, as an intuitive process, is experiencebased, fast and cognitively effortless and mental simulation, as a deliberative process, is slower and more cognitively demanding (see Klein, 1999). Feature matching is a dominant SA strategy (Kaempf et al., 1993) that can be viewed as an intuitive process, when a decision maker immediately recognizes the features, and as a deliberative process, when a decision maker consciously notices the features and uses them to retrieve a hypothesis or to contrast different hypotheses, concerning the particular unclear situation (Zsambok et al., 1992).

SA is usually followed by decision making (DM), which is defined as the selection of one option from a set of options (Klein, Calderwood, \& Clinton-Cirocco, 2010) and is interleaved with acting in complex realistic situations (Orasanu \& Conolly, 1993), requiring more than one decision. One way to make a decision is a straightforward identification of the situation along with an identification of the typical single option, which is usually automatically implemented. This experience-based, intuitive DM strategy, which corresponds with the RPD model, prevails in experienced people (Klein, 1999). However, when it is not clear what action to take, more cognitively demanding strategies are necessary. There are distinguished two types of option evaluation (Klein et al., 2010; Klein, Calderwood, \& MacGregor, 1989): sequential evaluation, when only one option is examined at a time, and concurrent evaluation, when several options are considered at the same time. The first one is characteristic for mental simulation, as the way of imagining the likely consequences of a single option, arriving at a satisficing decision. Notice that mental simulation is present in the RPD model as a supportive macrocognitive process (Crandall et al., 2006) of deliberative SA and DM. The second type of evaluation is characteristic for analytic $\mathrm{DM}$, as a way of comparing more options, arriving at an optimal decision. Flin, Youngson, and Yule (2007) write about creative and rule-based DM. Creative DM is viewed as devising a new option if certain unfamiliar problem or complication arises. Rule-based DM, typical for novices, involves remembering or looking up in a manual the option that applies. This concerns an evidence base or guidelines in medicine. I consider the non-intuitive strategies as deliberative.

The presented macrocognitive functions can be found in the list of paramedic non-technical skills (Shields \& Flin, 2012), which are fundamental for managing emergency medical services (EMS) ${ }^{1}$ situations. However, EMS physicians and paramedics have received less research attention in the NDM paradigm (e.g., Gurňáková et al., 2013; Smith et al., 2013) than other health professionals (e.g., Fackler et al., 2009; Pauley, Flin, Yule, \& Youngson, 2011). So I decided to investigate this field, while I am especially interested in EMS physicians, who are more competent than paramedics, and who are fully responsible for more severe cases del-

\footnotetext{
${ }^{1}$ In Slovakia and Czechia, there are currently two basic types of ground EMS ambulances with different staffing: ambulances led by physicians and ambulances without physicians usually led by more experienced paramedics.
} 
egated to them. Their main task is to stabilize the patient before transporting him/her to a hospital. The situations they encounter can be generally classified as routine or non-routine, which represent different situational factors that can affect the use of SA and DM strategies (Patel \& Arocha, 2001). According to the findings from other dynamic and time pressured fields, experienced professionals use mostly intuition (Klein, 1999). To my knowledge, this was empirically tested only in non-routine situations and a hypothesis was formulated only pertaining to higher frequency of intuition in routine situations (Crandall et al., 2006). Non-routine situations are the center of NDM research because they manifest accumulated experience and provide more specific information about cognition and expertise. Routine situations are discussed less. However, they are of some importance because perceiving many situations as routine suggests that professionals work in high-validity environment (i.e., stable relationships between cues and events/outcomes of actions) and have adequate opportunities for learning regularities of that environment (i.e., prolonged practice and rapid, unequivocal feedback) (Kahneman \& Klein, 2009). These are two sufficient conditions of expertise development that medicine meets.

The aim of this study is to identify which SA and DM strategies from the NDM perspective EMS physicians use in specific real-world routine and non-routine situations, and not which strategies from the personality perspective they use in general. I employed qualitative methodology to describe strategies and their context, which could be helpful in understanding the nature of these macrocognitive functions in the EMS field, and quantitative methodology to determine whether distribution of strategies is related to the type of situation. In accordance with the findings, I supposed that intuitive strategies will be used most frequently, while occurring more frequently in routine situations com- pared to non-routine situations. I also expected significant relationships between the types of situations and the types of strategies.

\section{Method}

\section{Participants}

The participants were 15 ground EMS physicians ( 7 males and 8 females) aged between 29 and $62(M=44.40, S D=10.20)$. All of them came from two states (11 from Slovakia and 4 from Czechia) and worked for different paramedic companies. Years of experience in health services varied from 4 to $37(M=18.87, S D=9.98)$ and years of experience in EMS varied from 3 to $37(M=15.73, S D=9.43)$, thus, none of the participants were novices. I supposed that professionals with at least three years of practice in the field have sufficient experience to perceive more situations as routine and, therefore, they are able to distinguish routine situations from non-routine situations. To recruit participants for this study, I contacted participants engaged in another research of EMS crews in which I had taken part before. I also used contacts provided by two physicians not interviewed in this research. All participants gave informed consent.

\section{Procedure}

For the study of non-routine situations I employed the Critical Decision Method (CDM; Klein et al., 1989), which is a retrospective semistructured in-depth interview method investigating critical or non-routine challenging situations from professionals' own practice. Interviewed professionals should be in a key decision-making role in the recalled situations. This NDM method was developed for eliciting knowledge, experience, and cognitive processes. The interviews were conducted in six steps described by Hoffman, Crandall, Klein, Jones, and 
Endsley (2008). At first, I instructed the interviewee to select and recall a situation. Then, I retold the situation to be sure of mutual understanding. During these steps, I was creating an initial timeline of the situation with decision points ${ }^{2}$, which I verified afterwards. For deepening I used a set of cognitive probes and "what if?" queries.

For the purpose of studying routine situations and comparing them with non-routine situations, I created an adapted and abbreviated version of the CDM. Although such adaptation was used for the first time, various adaptations with regard to research goals and possibilities are acknowledged in the literature (see Crandall et al., 2006). I reformulated the original instruction to recall a routine situation and omitted two steps: retelling the situation and "what if?" queries. I had two reasons for such abbreviation: first, my expectation of problems with conducting two long-lasting interviews (about routine and non-routine situation) in one session as well as in two sessions separated in time, and second, the omitted steps seemed redundant considering the research aim and lower cognitive demands of routine situations. So, both interviews took place in one session, and while the original version of CDM interview lasted 1.5 to 2 hours, the adapted version of CDM lasted approximately 30 minutes. This way I collected detailed descriptions of 15 routine and 15 non-routine situations.

To make routine and non-routine situations comparable within each participant, the participants had to recall situations, which were in some aspects similar but different in other aspects. I did not determine in what aspects the situations should differ in order to ensure subjective statements. The only exception (outside

${ }^{2}$ Decision points are points where a decision maker needed to assess a situation or make a decision, while there were different possible assessments and decisions/actions (Hoffman et al., 2008). the research scope) was a mass causality incident as a special case of EMS situations. The interview about a non-routine situation was intentionally and immediately followed by the interview about a routine situation. Since nonroutine situations happen very rarely, this sequence enabled the participants to find more easily comparable routine situations, which are many and varied. All the interviews were recorded on a digital voice recorder. After that, the participants received gift vouchers as a reward for research participation.

\section{Data analysis}

The audio recordings were transcribed and analyzed in the Atlast.ti software. I used the structured approach to CDM data (Wong, 2004) that lies in the analysis of decision points. Only the most important decision points for management of the situation were taken into account, not the obvious ones like taking the equipment to the patient or writing information about the patient. At first, I created a timeline-based decision chart of each situation (from the call from a dispatch center to transporting the patient to a hospital/leaving the patient at home) with decision points that served in subsequent situation summarization. Then, I created a decision analysis table, in which relevant cues, situation assessments, decisions, reasons, and goals were linked together. These stages of analysis are helpful for understanding the situation before analyzing items of interest in each decision point. By the described way I analyzed each situation separately, and finally, I summarized the decision points of all situations.

My items of interest were codes for four topics. Two topics were related to the following strategies: types of intuitive and deliberative SA strategies (pattern matching, feature matching, mental simulation) and types of intuitive and deliberative DM strategies (intuitive, mental simulation, analytic, creative, rule-based). 
Definitions of strategies are the same as the definitions in the introduction section. Two more topics were related to situations: types of situations (routine, non-routine) and types of situational factors emerged in previous yet unpublished analysis of the interviews (patient's medical condition, patient's behavioral and psychological characteristics, physical environment, other people's behavioral and psychological characteristics). The coding process was controlled by discussing ambiguous statements with the second rater in order to reach an agreement. Following qualitative analysis, statistical analyses were performed in SPSS to determine whether there are associations between the variables.

\section{Results}

Overall, 93 decision points involving SA (ranged from 1 to 9 with an average of 3 in each situation) and 172 decision points involving DM (ranged from 2 to 15 with an average of 6 in each situation) were analyzed, while each decision point was about one of the four situational factors. The largest number of decision points in routine and non-routine situations was about the patient's medical condition ( 88 related to SA, 164 related to DM). Only in non-routine situations, the decisions points were also about patient's behavioral and psychological characteristics (1 related to $\mathrm{SA}, 1$ related to $\mathrm{DM})$, physical environment ( 2 related to SA, 3 related to DM), and other people's behavioral and psychological characteristics (2 related to SA, 4 related to $\mathrm{DM}$ ).

Illustrative quotations of SA strategies are given in Table 1, and the distribution of SA strategies within the types of situations is presented in Table 2. In all routine situations $(100 \%)$ but also in many non-routine situations $(85 \%)$, the most frequently used strategy was intuitive SA, as I called it, due to the difficulty of determining whether SA was developed by pattern matching (seen as intuitive strategy) or feature matching (used intuitively).

The decision points handled using deliberative feature matching (12\%) and mental simulation $(3 \%)$ were only in four non-routine situations and were related to patient's medical condition. More specifically, only these SAs were related to patient's ambiguous health problems.

Table 1 Examples of quotations illustrating SA strategies

\begin{tabular}{ll}
\hline SA strategy & Quotation \\
\hline Intuitive & "I found out that airways are free, breathing is smooth, that it's not \\
& rattling, but definitely he is deeply unconscious. Looking at his \\
& face, his eye pupils, I could immediately see that this was intra- \\
& cranial or brain injury." \\
Deliberative feature & "...the baby looks normal or it is maceration as described in books, \\
matching & that if the fetus is dead, the skin is macerated. So, that was the \\
& problem for me. Is it macerated or is it not macerated? ... I \\
& examined it and macerated skin should be rather softened, and so I \\
& said that's probably the case." \\
& "...when he [the gastroenterologist] did the colonoscopy I was \\
& thinking about what was going on, how much air he insufflated \\
there, if he took histology from one place, from two places, how \\
the intestinal mucosa looked, that it was probably fragile, that it \\
ruptured."
\end{tabular}


Table 2 Distribution of types of SA strategies within types of situations

\begin{tabular}{lccccc}
\hline SA strategy & & Non-routine situation & \multicolumn{2}{c}{ Routine situation } \\
\hline Intuitive & & 49 & $85 \%$ & 35 & $100 \%$ \\
\multirow{3}{*}{ Deliberative } & Feature matching & 7 & $12 \%$ & - & - \\
$\Sigma$ & Mental simulation & 2 & $3 \%$ & - & - \\
$\Sigma$ & & 58 & $100 \%$ & 35 & $100 \%$ \\
\hline
\end{tabular}

In two such situations, previous experience suggested that the health problem is not like the health problem seen before. Although the participants had no direct experience with these cases until then, their medical knowledge enabled them to reach some SA. In two other such situations, previous experience suggested various more and less serious diagnoses seen before at least once and it helped the participant to exclude the least likely diagnoses step by step and reach a final SA. One to five hypotheses concerning the diagnoses were generated and evaluated. These were related to either understanding the observed signs and symptoms by feature matching and/or understanding the causes of health problems by mental simulation. In two of these four situations, EMS physicians had two competing, almost equally important tasks at the same time: to assess (diagnose) the causes of health problems and to assess the patient's rapidly changing medical condition for the purpose of stabilization. In all other situations, one task was always more important, usually to assess the patient's medical condition for stabilization.

In sum, in non-routine situations about $85 \%$ of assessments were developed intuitively and $15 \%$ deliberately, whereas in routine situations $100 \%$ of assessments were developed intuitively. To test the association between two types of situations (routine or non-routine) and two main types of SA strategies (intuitive or deliberative) with one sampling zero (i.e., zero count of deliberative SA strategy in routine situations), Fisher's exact test ${ }^{3}$ was run and a small constant (0.5) was added to each cell to calculate the odds ratio. The test showed significant association, $p=.012$. Based on the odds ratio, using intuitive SA strategies compared to deliberative SA strategies was $1 / 0.073=13.70$ times more likely in routine situations than in nonroutine situations.

Illustrative quotations of DM strategies are given in Table 3, and the distribution of DM strategies according to the types of situations is presented in Table 4. Intuitive DM was the most frequently used strategy in non-routine situations $(83 \%)$ as well as in routine situations (93\%). It was used in each situation at least once. Intuitive decisions were accompanied by the automatic implementation of the first experience-based option that came to mind. Deliberate DM strategies were used in three routine situations and in 12 non-routine situations, in which decision points were related to various situational factors. Only in non-routine situations, the participants mentally simulated (3\%) hypothetical consequences of each identified option and evaluated their plausibility in terms of risks for the patient. In two decision points, they simulated a single option with which they had not had enough experience before, and in

\footnotetext{
${ }^{3}$ In this and subsequent categorical data analysis, each decision point contributed to only one cell of the table and was considered independent.
} 
one decision point they simulated two wellknown options. The simulated options were either implemented or rejected. The decision points handled by analytic DM strategy appeared less in routine situations $(7 \%)$ in contrast with non-routine situations (11\%), while only two options were compared. In ten deci- sion points, the participants compared the options only briefly with regards to patient' health benefits. But in non-routine situations a need for justification emerged in one decision, asking for an advice from the EMS team member emerged in another decision, and four analytical decisions were based on adopting an ad-

Table 3 Examples of quotations illustrating DM strategies

\begin{tabular}{ll}
\hline DM strategy & Quotation \\
\hline Intuitive & "...vein on the baby's head was pricked but its patency wasn't \\
& sufficient or it was burst or something like that. Then we auto- \\
& matically used the intraosseous access. But I didn't consider going \\
ahead or not going ahead." & "I imagined that something happens in the head [stroke, brain \\
Mental simulation & tumor, or any other serious disease], I'll leave him at home and \\
& he'll fall unconscious and die by the morning. Once I had the \\
& thought that there is any likelihood that this scenario could happen, \\
& then I couldn't leave him at home." \\
& "I was deciding whether to administer clear saline or saline with \\
Analytic & noradrenalin. ... Considering that the patient had somewhat lower \\
& blood pressure but he had a good pulse and good saturation, I \\
& decided to administer saline with noradrenaline." \\
& "Draining both sides of the chest, it doesn't happen very routinely. \\
And we had to improvise a lot because actually there was only one & \\
chest drain kit. ... Actually, from one side we used the chest drain & kit we had and from the other side we used a thin intubation \\
cannula primarily made for securing of the airways, so we used it as & a chest drain."
\end{tabular}

Table 4 Distribution of types of DM strategies within types of situations

\begin{tabular}{llcccc}
\hline DM strategy & & Non-routine situation & \multicolumn{2}{c}{ Routine situation } \\
\hline Intuitive & & 82 & $83 \%$ & 68 & $93 \%$ \\
& Mental simulation & 3 & $3 \%$ & - & - \\
\multirow{3}{*}{ Deliberative } & Analytic & 11 & $11 \%$ & 5 & $7 \%$ \\
& Creative & 3 & $3 \%$ & - & - \\
$\Sigma$ & Rule-based & - & - & - & - \\
& & 99 & $100 \%$ & 73 & $100 \%$ \\
\hline
\end{tabular}


vice/a recommendation from other professionals (operator in the dispatch center, fireman, physician in the specialized health care center) or a request from the patient's relative. Creative DM strategy was needed in few decision points (3\%) of non-routine situations, in which EMS physicians created only one good enough option for implementation. Options that had never been used or considered before were devised with the help of previous experience and knowledge, making it possible to recognize opportunities to overcome complications. Neither decision point was coded as rule-based DM.

In sum, in non-routine situations about $83 \%$ of decisions were made intuitively and $17 \%$ deliberately, whereas in routine situations about $93 \%$ of decisions were made intuitively and $7 \%$ deliberately. To test the association between two types of situations (routine or non-routine) and two main types of DM strategies (intuitive or deliberative), Pearson's chi-square test was run, showing significant association, $\chi^{2}(1)=$ $4.014, p=.045$. This represent the fact that, based on the odds ratio, using intuitive DM strategies compared to deliberative DM strategies was $1 / 0.355=2.82$ times more likely in routine situation than in non-routine situations.

Due to wide range of EMS physicians' biographical data (age, years of experience in health services, years of experience in EMS) and their possible relationships with the frequency of each strategy used (eight frequencies related to each combination of SA or DM, intuitive or deliberative strategy, routine or nonroutine situation), I performed correlation analyses that showed no significant relationships, $r_{s}$ varied from -.268 to $.159, p$ varied from .335 to .972 .

\section{Discussion}

The analysis revealed that EMS physicians had to deal mainly with the patient's medical condition but in non-routine situations also with other situational factors, such as the patient's behavioral and psychological characteristics, the physical environment, and the other people's behavioral and psychological characteristics. Some of the factors or problems can be found in the study by Harenčárová (2017), but she did not categorize them as I did. The quantitative part of the results met my expectations about the prevalent use of intuition and the association of strategies with situations. The intuitive SA and DM strategies, compared to deliberative SA and DM strategies, prevailed in both types of situations, specifically, they prevailed more in routine situations compared to non-routine situations. Such massive use of intuition provides empirical support for the RPD model (Klein, 1999) and emphasizes the value of previous experience. Looking at the different odds ratios, using intuitive SA strategies in routine situations was more likely than using intuitive DM strategies in routine situations. In other words, the routine and non-routine situation category was more strongly related to SA strategies than to DM strategies. Maybe such category (included in the CDM instruction) was too general and, therefore, EMS physicians recalled situations of very different nature. It may be insufficient in the case of searching for relationships with strategies. Thus, introducing more specification would be more appropriate in the future (e.g., distinguishing routine and non-routine situations from the point of view of SA, DM, and/or situational factors). For comparison, surgeons' decision making process has not been shown to be related to situational factors (type of operation - endoscopic or open, context - emergency or elective surgery, time pressure, situational threat) (Pauley et al., 2011). The interpretation is that the researchers studied only one decision point in each surgeon's situation, compared to more decision points studied in each EMS physician's situation. The nature of the tasks and situational factors during surgery definitely differ from EMS. EMS is 
a more dynamic, time pressured field involving the above mentioned and other situational factors.

To be specific, EMS physicians most frequently used intuitive SA strategy in both types of situations. Thus, the assessments were clear at the moment during these situations. But from the transcripts it was difficult to distinguish whether the intuitive SAs were carried out by pattern matching as an intuitive process (see Klein, 1999) or by feature matching, which can also be seen as an intuitive process (see Zsambok et al., 1992). Maybe in this case, pattern and set of features were somehow linked together. Although I did not find any other details about the nature of intuitive SA, it can be based on either unique highly context specific patterns (more common) or more general classic complete patterns (Fackler et al., 2009).

In four non-routine situations with the patient's ambiguous health problems, EMS physicians developed some SAs by deliberative use of feature matching (to understand signs and symptoms) and mental simulation (to understand the causes of health problems). The ambiguity is the reason for using these two strategies and then the situations become clearer (Klein, 1999). It means that although EMS physicians encountered such situations for the first time, they were able to develop assessments because no situation was out of their professional field (medicine). Compared to feature matching, they used mental simulation less often, same as Kaempf et al. (1993) found in AEGIS personnel. Note that their study included the strategy of story building (Pennington \& Hastie, 1993), which is conceptually equivalent to mental simulation. The findings about SA strategies are close to the findings of Patel and Arocha (2001), who state that if the nature of a health problem is known, physicians use forward reasoning (from signs and symptoms to diagnosis). On the contrary, if the nature of a health problem is unknown, experi- enced physicians use backward reasoning (from generating possible diagnoses to signs and symptoms), which makes heavy demands on working memory, or rather calls for mixed strategy to deal with known and unknown aspects of the problem.

Focusing on the distribution of DM strategies, EMS physicians predominantly made intuitive decisions in both types of situations. Making many intuitive decisions is typical also for other dynamic, time pressured fields like firefighting (Klein et al., 2010), Navy (Kaempf et al., 1993), or sport (Macquet, 2009), in which there is often not enough time for analyzing multiple options.

EMS physicians few times mentally simulated options in non-routine situations to determine whether they are satisficing in terms of risks. The satisficing principle (selecting the first option that meets the criteria) characteristic for NDM is linked to the research of Simon (1955). The physicians employed analytical DM several times in both types of situations but more frequently in the non-routine ones. The way they made some analytical decisions can be qualified as noncompensatory (Zsambok et al., 1992), because they did not analyze if positive attributes compensated for negative ones, but they focused solely upon positive attributes: option benefits and adequacy for the patient's medical condition. This is a simpler way of DM in contrast to their decisions where other people were involved in some manner (expected justification, advice, recommendation, or request). However, more time spent thinking through the options and their consequences occurs more likely in surgeons, who encounter situations that require fewer immediate actions (Pauley et al., 2011).

In some non-routine situations, EMS physicians also needed to make creative decisions they had never made before. Despite situational problems, they were able to improvise and use opportunities, the identification of which was 
facilitated by their experience and knowledge base. Consequently, they did not have to search for a solution too long. In my opinion, their knowledge base made them trust the new options. Such identification of opportunities and turning them into an option is one of the supporting macrocognitive processes (Crandall et al., 2006; Klein et al., 2003).

Failure to identify the rule-based decisions does not necessary mean that no rule had been followed. There are guidelines for EMS personnel representing a set of context free rules or recommendations based on scientific proof (see Rosenberg \& Donald, 1995). But these rules can merge with experience or become automatic and can be retrieved from memory with little conscious effort, i.e. intuitively or recognitionprimed (Flin et al., 2007). This fact is the rationale for the result, because most EMS physicians worked many years in the domain. Moreover, completely following the rules may sometimes be difficult in context specific situations (specificity of patient's medical condition and circumstances).

The probable relationships between the EMS physicians' years of experience and the numbers of used strategies have not been demonstrated. Maybe examining experiment-based, standardized situations reducing the uniqueness of each situation, or including novices (without much previous experience) in the research sample, who make less intuitive and more analytical decisions (Klein, 1999), would bring different findings. Nevertheless, experience and its relation to effective acting should be discussed. According to the conflict theory (Mann et al., 1997), in which definitions of vigilance and hypervigilance are compatible with definitions of analysis and intuition, respectively, vigilance is seen as the only adaptive and effective decision strategy. But Johnston, Driskell, and Salas (1997) assert that hypervigilance can be more effective than vigilance, if a decision maker is an expert under time pressure. This is exactly what the NDM research found about intuitive decisions (Klein, 1997). However, from this perspective I cannot state how effectively EMS physicians acted in the recalled situations, but they made many experience-based decisions when it comes to the frequency of intuitive strategies used. And because years of experience do not automatically guarantee intuitive expertise (Kahneman \& Klein, 2009), I cannot even state whether they were experts. But they were more and less experienced professionals when it comes to the years of experience in the field.

In this study, uncertainty that affects SA and DM (Rimstad \& Sollid, 2015) was not investigated. Types and intensity of uncertainty could probably be significant in this sense. Harenčárová (2017) found that the paramedics' situation phase predicted uncertainty regarding inadequate understanding of the situation, action, cause, and outcome, requiring engaging coping strategies defined by the RAWFS heuristic (Lipshitz, Omodei, McClellan, \& Wearing, 2007). I suppose that EMS physicians experienced uncertainty particularly in non-routine situations as suggested by the use of deliberative strategies, which may be considered a form of coping strategies. Another relevant finding is the demonstration of predictive value of personality for decision preferences in health professionals, including paramedics (e.g., Gurňáková et al., 2013; Pilárik \& SarmánySchuller, 2011). But I think to take the personsituation interaction into account would be more beneficial. The NDM seems to be a promising approach for addressing this issue.

In conclusion, I realize some limitations such as small sample size and retrospective subjective data, hence the results should be taken carefully. Be that as it may, this investigation verified some aspects of the RPD model in two types of situations, but especially, it brought insight into SA and DM strategies, which have not been studied very much within the EMS field (see Shields \& Flin, 2012). The studied EMS 
physicians prevalently used cognitively simpler intuitive strategies in routine and non-routine situations, and they used cognitively more challenging deliberative strategies less, mainly in routine situations. The association of strategies with situations suggests that EMS physicians tended to change the strategies with respect to situational demands. These findings highlight the importance of situational aspect and the importance of intuition and deliberation. This should be kept in mind in assessing and improving the quality of EMS professionals' performance. Since better understanding of the discussed topics is still needed, I encourage researchers to study them further.

\section{References}

Crandall, B., Klein, G., \& Hoffman, R. R. (2006). Working minds: A practitioner's guide to cognitive task analysis. Cambridge, MA: MIT Press.

Evans, J. St. B. T. (2007). Hypothetical thinking: Dual processes in reasoning and judgement. Hove, East Sussex: Psychology Press.

Fackler, J. C., Watts, C., Grome, A., Miller, T., Crandall, B., \& Pronovost, P. (2009). Critical care physicians cognitive task analysis: An exploratory study. Critical Care, 13:R33.

Flin, R., Youngson, G., \& Yule, S. (2007). How do surgeons make intraoperative decisions? Quality and Safety in Health Care, 16, 235-239.

Gurńáková, J., et al. (2013). Rozhodovanie profesionálov: Sebaregulácia, stres a osobnost'. Bratislava: Ústav experimentálnej psychológie SAV.

Harenčárová, H. (2017). Managing uncertainty in paramedics' decision making. Journal of Cognitive Engineering and Decision Making, 11, 42-62.

Hoffman, R. R., Crandall, B., Klein, G., Jones, D. G., \& Endsley, M. R. (2008). Protocols for cognitive task analysis. Pensacola, FL: Institute for Human \& Machine Cognition.

Johnston, J. H., Driskell, J. E., \& Salas, E. (1997). Vigilant and hypervigilant decision making, Journal of Applied Psychology, 82, 614-622.

Kahneman, D., \& Klein, G. (2009). Conditions for intuitive expertise: A failure to disagree. American Psychologist, 64, 515-526.

Kaempf, G. L., Wolf, S., \& Miller, T. E. (1993). Decision making in the AEGIS combat information center. In Proceedings of the Human Factors and Er- gonomics Society 37th Annual Meeting (pp. 11071111). Santa Monica, CA: Human Factors and Ergonomics Society.

Klein, G. (1997). The current status of the naturalistic decision making framework. In R. Flin, E. Salas, M. Strub, \& L. Martin (Eds.), Decision making under stress: Emerging themes and applications (pp. 1128). Farnham: Ashgate Publishing Limited.

Klein, G., Calderwood, R., \& Clinton-Cirocco, A. (2010). Rapid decision making on the fire ground: The original study plus a postscript. Journal of Cognitive Engineering and Decision Making, 4(3), 186209.

Klein, G. A. (1999). Sources of power: How people make decisions. Cambridge, Massachusetts: The MIT Press.

Klein, G. A., Calderwood, R., \& MacGregor, D. (1989). Critical decision method for eliciting knowledge. IEEE Transactions on Systems, Man, and Cybernetics, 19, 462-472.

Klein, G. A., Ross, K. G., Moon, B. M., Klein, D. E., Hoffman, R. R., \& Hollnagel, E. (2003). Macrocognition. IEEE Intelligent Systems, 18(3), 81-85.

Lipshitz, R., Omodei, M., McClellan, J., \& Wearing, A. (2007). What's burning? The RAWFS heuristic on the fire ground. In R. R. Hoffman (Ed.), Expertise out of context: Proceedings of the sixth international conference on naturalistic decision making (pp. $97-$ 111). Mahwah, NJ: Lawrence Erlbaum Associates.

Macquet, A. C. (2009). Recognition within the decision making process: A case study of expert volleyball players. Journal of Applied Sport Psychology 21, 64-79.

Mann, L., Burnett, P., Radford, M., \& Ford, S. (1997). The Melbourne Decision Making Questionnaire: An instrument for measuring patterns for coping with decisional conflict. Journal of Behavioral Decision Making, 10, 1-19.

Orasanu, J., \& Connolly, T. (1993). The reinvention of decision making. In G. A. Klein, J. Orasanu, R. Calderwood, \& C. E. Zsambok (Eds.), Decision making in action: Models and methods (pp. 3-20). New Jersey: Ablex.

Patel, V. L., \& Arocha, J. F. (2001). The nature of constraints on collaborative decision making in health settings. In E. Salas, \& G. A., Klein (Eds.), Linking expertise and naturalistic decision making (pp. 383406). Mahwah, NJ: Lawrence Erlbaum Associates.

Pauley, K., Flin, R., Yule, S., \& Youngson, G. (2011). Surgeons' intraoperative decision making and risky management. The American Journal of Surgery, 202, 375-381.

Pennington, N., \& Hastie, R. (1993). A theory of explanation-based decision making. In G. A. Klein, J. 
Orasanu, R. Calderwood, \& C. E. Zsambok (Eds.), Decision making in action: Models and methods (pp. 188-201). Norwood, CT: Ablex.

Pilárik, L., \& Sarmány-Schuller, I. (2011). Personality predictors of decision-making of medical rescuers. Studia Psychologica, 53, 175-184.

Rimstad, R., \& Sollid, S. J. M. (2015). A retrospective observational study of medical incident command and decision-making in the 2011 Oslo bombing. Journal of Emergency Medicine, 8(4), 1-10.

Rosenberg, W., \& Donald, A. (1995). Evidence based medicine: An approach to clinical problem-solving. British Medical Journal, 310, 1122-1126.

Shields, A., \& Flin, R. (2012). Paramedics' non-technical skills: A literature review. Emergency Medicine Journal, 30, 350-354.

Simon, H. A. (1955). A behavioral model of rational choice. Quarterly Journal of Economics, 69, 99 118 .
Simon, H. A. (1992). What is an „explanation“ of behavior? Psychological Science, 3, 150-161.

Smith, M. W., Bentley, M. A., Fernandez, A. R., Gibson, G., Schweikhart, S. B., \& Woods, D. D. (2013). Performance of experienced versus less experienced paramedics in managing challenging scenarios: A cognitive task analysis study. Annals of Emergency Medicine, 62, 367-379.

Wong, B. L. W. (2004). Critical decision method data analysis. In D. Diaper, \& N.A. Stanton (Eds.), The Handbook of task analysis for human computers interaction (pp. 327-346). Mahwah, New Jersey: Lawrence Erlbaum Associates.

Zsambok, C. E., Beach, L. L., \& Klein, G. (1992). A literature review of analytical and naturalistic decision making. (Task 2: Final technical report prepared under contract N66001-90-C-6023 for the Naval Command, Control and Surveillance Center, San Diego, CA). Fairborn, OH: Klein Associates Inc. 